\title{
miR-1246 shuttling from fibroblasts promotes colorectal cancer cell migration
}

\author{
Guifan $\mathrm{SI}^{1}$, Suyun $\mathrm{LI}^{2}$, Qi ZHENG ${ }^{3}$, Shiguo ZHU ${ }^{1, \star}$, Chunxian ZHOU ${ }^{2, *}$ \\ ${ }^{1}$ Laboratory of Integrative Medicine, School of Basic Medical Sciences, Shanghai University of Traditional Chinese Medicine, Shanghai, China; \\ ${ }^{2}$ Department of Pathology, School of Basic Medical Sciences, Shanghai University of Traditional Chinese Medicine, Shanghai, China; ${ }^{3}$ Depart- \\ ment of Pathology, School of Basic Medical Sciences, Fudan University, Shanghai, China
}

${ }^{*}$ Correspondence: cxzhou@shutcm.edu.cn; zhushiguo@shutcm.edu.cn

Received September 24, 2020 / Accepted November 11, 2020

\begin{abstract}
Cancer-associated fibroblasts (CAFs) are the major constituents of the tumor microenvironment and promote cancer development via tumor-stromal interactions. The alteration of microRNA (miRNA) expression in fibroblasts can induce the phenotype conversion between normal fibroblasts and CAFs in certain tumor types. However, the mechanisms underlying the phenotype conversion of fibroblasts in colorectal cancer (CRC) are largely unknown. Our study focuses on the role of miR-1246 in fibroblasts-CRC cells interaction. In this study, CCD-18Co colorectal fibroblasts were cultured in the conditioned medium (CM) derived from CRC cells to obtain the CAF phenotype. We found that the miR-1246 expression was upregulated in CAF-like fibroblasts compared with normal fibroblasts. miR-1246 secreted by cancer cells could be utilized by neighboring fibroblasts for CAF reprogramming. On the other hand, following secretion by CAF-like fibroblasts, miR-1246 was delivered into CRC cells and promoted cell migration via the activation of the Wnt/ $\beta$-catenin signaling in CRC cells. Furthermore, high miR-1246 expression in CRC tissues was negatively associated with disease-free survival (DFS) for CRC patients. Taken together, our results reveal that miR-1246 can shuttle between CRC cells and fibroblasts. This study also indicates that targeting miR-1246 or blocking its transport from CAFs to CRC cells might represent a novel therapeutic approach in CRC treatment.
\end{abstract}

Key words: fibroblast, miR-1246, colorectal cancer, disease-free survival

Fibroblasts in the tumor microenvironment that are highly activated are called cancer-associated fibroblasts (CAFs). The progression of tumors is not only determined by cancer cells but also promoted by CAFs [1]. Previous studies report that fibroblasts can stimulate malignant properties of cancer cells, such as cell proliferation, cell motility, and angiogenesis [2, 3 ]. The crosstalk between cancer cells and stromal fibroblasts has already been described [4]. Cancer cells can activate fibroblasts and induce their transdifferentiation into CAFs. On the other hand, CAFs promote cancer progression [5]. Abnormal gene expression or non-coding RNA levels have impacts on fibroblasts' transdifferentiation. For example, HIPK2 knockdown in fibroblasts induces a CAF-like phenotype [5]. However, the mechanism of the phenotype conversion between normal fibroblasts (NFs) and CAFs is still elusive in CRCs.

MicroRNAs (miRNAs) are small non-coding RNAs consisting of approximately 23 nucleotides, which play a key role in the negative regulation of target genes expres- sion [6]. The alteration of miRNA expression in fibroblasts can induce phenotype conversion between NF and CAF. For example, modifying the expression of miR-1, miR-206, and miR-31 in lung fibroblasts induces a functional conversion between NF and CAF [7]. In addition, the expression of miR-31, miR-214, and miR-155 is deregulated in ovarian CAFs. Mimicking the deregulation of these miRNAs has been shown to induce functional conversion between NF and CAF [8]. Moreover, miR-222 is upregulated in breast cancer CAFs and plays a key role in controlling fibroblast phenotypes [9]. However, whether miRNAs exist to regulate the phenotype conversion of fibroblasts in CRC remains unknown.

In this study, we found miR-1246 could shuttle between cancer cells and fibroblasts. We observed that miR-1246 from cancer cells induced fibroblasts reprogramming to the CAF-like phenotype. Besides, uptake of miR-1246 derived from fibroblasts by colon cancer cells promoted cell migration via regulation of the Wnt/ $\beta$-catenin signaling pathway. 


\section{Materials and methods}

Cell culture and miRNA transfection. CCD-18Co human colorectal fibroblasts were sourced from ATCC (CRL-1459) and were kindly provided by Dr. Shuyang Wang. Cells were cultured in Eagle's Minimum Essential Medium (EMEM) containing 10\% FBS (Gibco). DLD-1 and HCT-8 cells were obtained from Shanghai Institute of Cell Biology, Chinese Academy of Sciences (TCHu134 and TCHu 18), and were grown in RPMI 1640 medium containing 10\% FBS. miR-1246 mimics, inhibitors, and negative controls (NC) were transfected to cells using Lipofectamine RNAiMAX Reagent (Invitrogen).

Conditioned medium (CM). The media were changed to serum-free media when colon cancer cells (DLD-1, HCT-8, Lovo, and HCT-116) were sub-confluent. The media were harvested $48 \mathrm{~h}$ later and centrifuged at $4000 \times \mathrm{g}$ for $10 \mathrm{~min}$, to collect the supernatant as the CM. CMs from CCD-18Co cells were generated by adding fresh media to cells of $>50 \%$ confluence. The media were collected $48 \mathrm{~h}$ later and centrifuged at $4000 \times \mathrm{g}$ for $10 \mathrm{~min}$ to collect the supernatant. To avoid contamination of transfection regent/miRNA complex in $\mathrm{CM}$, the culture media of cells that were transfected with NC or miR-1246 mimics were changed $5 \mathrm{~h}$ after transfection with fresh media. Supernatants were collected as described.

Microarray hybridization. CCD-18Co, CCD-18Co treated with DLD-1 CM, and HCT-8 CM were sent to Shanghai Biotechnology Corporation for microarray analysis by Agilent human miRNA $(8 \times 60 \mathrm{~K}) \mathrm{V} 21.0$. Briefly, the RNA sample was labeled with the fluorescent dye Cy3. The labeling and hybridization were performed using miRNA Complete Labeling and Hyb Kit (Agilent Technologies) according to the manufacturer's instructions. Slides were scanned by Agilent Microarray Scanner installed with Feature Extraction Software 10.7 (Agilent technologies) using default settings. Raw data were normalized by R package AgiMicroRna using Quantile algorithm [10].

RNA extraction and quantitative RT-PCR (qRT-PCR). Total RNA was extracted using Trizol reagent according to the manufacturer's instructions (Invitrogen). miRNA expression levels were measured by qRT-PCR using Taqman MicroRNA Assays (Applied Biosystems). The expression levels of U47 small nuclear RNA were used as endogenous controls for miRNA. Data were analyzed using $2^{\Delta \Delta \mathrm{Cq}}$ method.

Immunofluorescence. Cells were seeded in a 6-well culture plate containing a glass coverslip in each well. After treatment, cells were fixed with $4 \%$ paraformaldehyde, washed with PBS, and permeabilized in $0.1 \%$ Triton X-100. After blocking with $3 \%$ horse serum for one hour, cells were incubated with anti-aSMA antibody (1:250, Sigma, A5228), $\beta$-catenin (1:500, Proteintech, 51067-2-AP) for $2 \mathrm{~h}$, followed by incubation with Cy3-conjugated secondary antibody (Proteintech) or Alexa Fluor 488 Conjugated anti-rabbit antibody (Cell Signaling Technology) for $1 \mathrm{~h}$. DAPI was used for nuclear counterstaining. The coverslips were observed under a confocal microscope at $400 \times$ magnification (Leica, Microsystems).

Cell growth and migration. Cell growth and migration assays were performed as described previously [11]. Briefly, DLD- 1 and HCT- 8 cells were cultured in 96-well plates. After $24 \mathrm{~h}$, cells were treated with CM from CCD-18Co cultures previously transfected with NC or miR-1246 mimics. Cell numbers were measured using a Cell Counting Kit, $24 \mathrm{~h}$ later. Cell migration assays were performed using transwell migration chambers ( $8 \mu \mathrm{m}$, Corning). DLD-1 and HCT- 8 cells, which were pretreated with $\mathrm{CM}$, were loaded to the upper chamber in a medium containing 1\% FBS. Medium containing $10 \%$ FBS was added in the lower chamber. Cells that migrated into the lower chamber were stained with $0.1 \%$ crystal violet and counted.

Indirect co-culture of CCD-18Co and DLD-1 cells. Indirect co-culture was performed using a transwell system with $0.4 \mu \mathrm{m}$ pore membranes. GFP-labeled DLD- 1 cells were cultured on a coverslip in the lower chamber. CCD-18Co cells were transfected with Cy3-tagged miR-1246 and cultured on the surface with a $0.4 \mu \mathrm{m}$ pore insert. Media were changed 5 h later with or without GW4869, and then the cells were cultured with GFP-labeled DLD-1 cells for $20 \mathrm{~h}$. The coverslips were observed under a confocal microscope at $630 \times$ magnification.

Western blot. Proteins were harvested with Rapa lysis buffer, resolved by SDS-PAGE, and transferred to nitrocellulose membranes. After blocking with 5\% BSA, the membrane was incubated with the primary antibody, followed by the secondary antibody. Finally, the membrane was visualized by chemiluminescence. The following antibodies were used: aSMA (1:1000, Sigma, A5228), Vimentin (1:1000, Cell Signaling Technology, 5741S), $\beta$-catenin (1:1200, Cell Signaling Technology, 8480S), AXIN2 (1:1000, Cell Signaling Technology, 2151S), and GSK3 $\beta$ (1:2000, Cell Signaling Technology, 12456T).

Survival analysis. miR-1246 expression level in CRC tissues was obtained from the TCGA database. Kaplan-Meier survival analysis was performed to observe the survival rate of miR-1246 by Medcalc software. Associations between gene expression and disease-free survival (DFS) or overall survival (OS) were quantified by hazard ratio (HR) and corresponding 95\% CIs from Cox proportional hazards regression.

\section{Results}

CRC cells upregulate miR-1246 level in fibroblasts and induce phenotype conversion of fibroblasts. Normal colorectal fibroblasts CCD-18Co were cultured in conditioned medium (CM) from colorectal cancer cell DLD-1 or HCT-8 cells. The cells developed a CAF-like phenotype, leading to the upregulation of aSMA, the marker of activated fibroblasts [7] (Figure 1A). By comparison with the miRNA levels in CCD-18Co using miRNA microarrays, we observed that four miRNAs were three-fold higher in their expression 

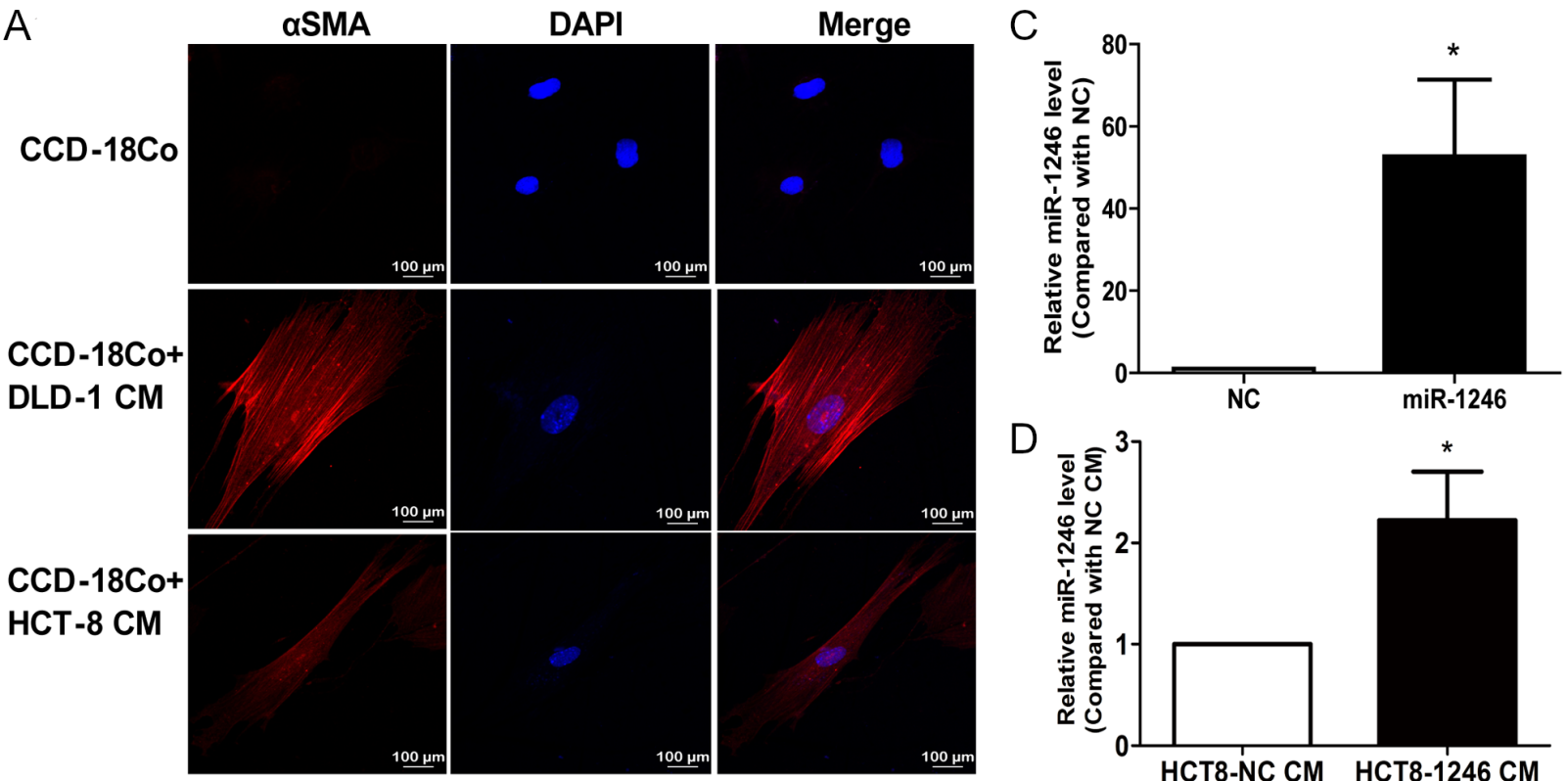

$\mathrm{B}$
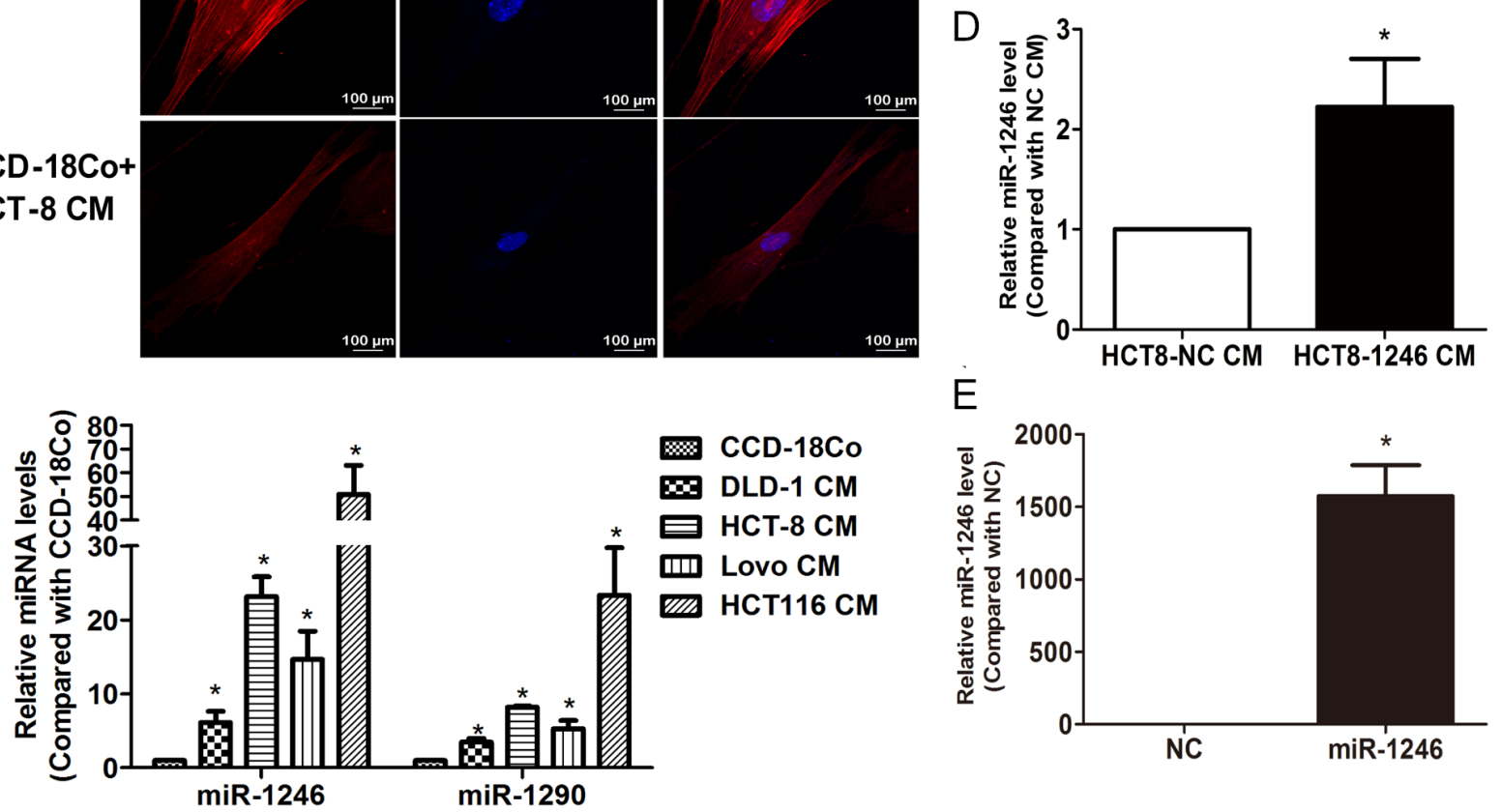

G

$\mathrm{F}$
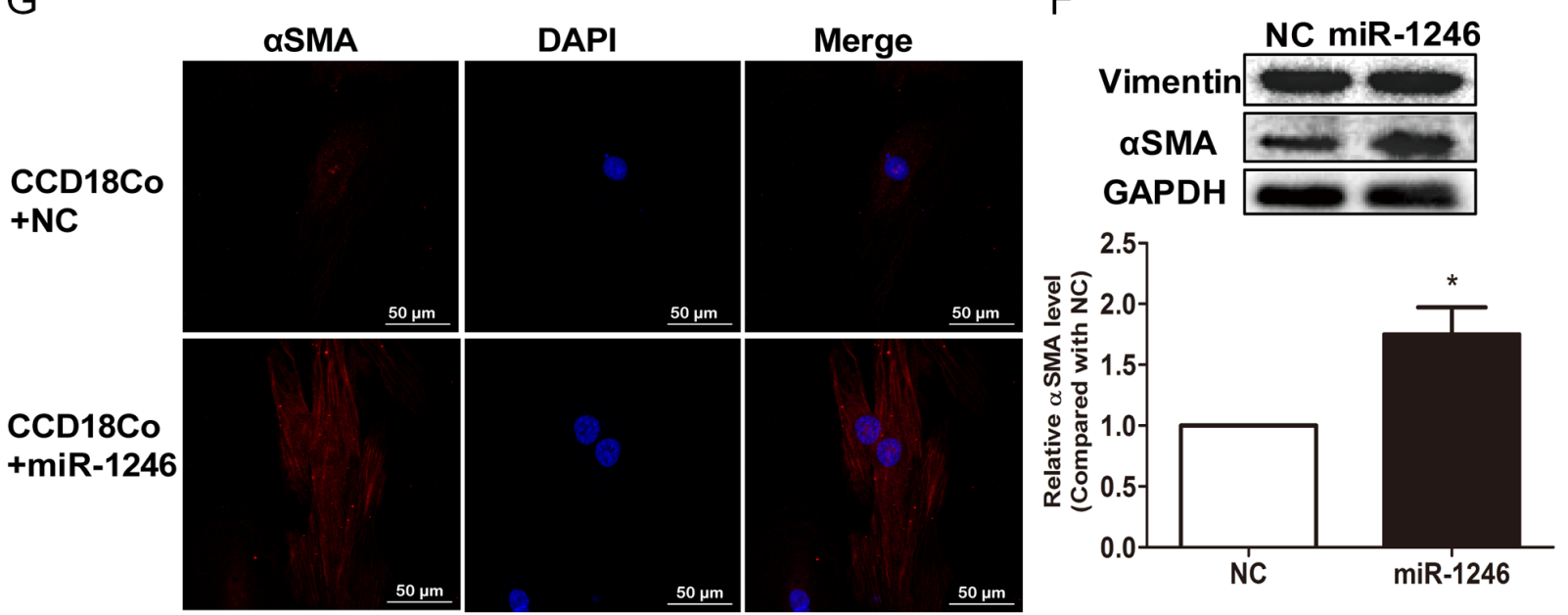

Figure 1. miR-1246 was overexpressed in CAF-like fibroblasts and induced phenotype conversion of fibroblasts. A) Immunofluorescence analyses of aSMA expression in CCD-18Co cells which was cultured in CM from DLD-1 and HCT-8 cells. B) CCD-18Co cells were cultured in CM from four CRC cell lines (DLD-1, HCT-8, Lovo, and HCT-116). qRT-PCR analyses of miRNA expression in CCD-18Co cells. ${ }^{*}$ p $<0.05$ compared with miR-1246 level in CCD-18Co. C) miR-1246 expression was increased in HCT-8 cells transfected with miR-1246 mimics. ${ }^{*} p<0.05$ compared with miR-1246 levels in the NC group. D) CCD-18Co cells were cultured in the CM from HCT-8 with overexpression of miR-1246, $q$ RT-PCR analyzes the expression level of miR-1246 in CCD-18Co. ${ }^{*} p<0.05$ compared with $\mathrm{CM}$ from the NC group. E) miR-1246 was overexpressed after miR-1246 mimics were transfected into CCD-18Co. Western blot $(F)$ and immunofluorescence $(G)$ showed CCD-18Co cells transfected with miR-1246 mimics had increased aSMA level compared with cells transfected with NC. ${ }^{*} \mathrm{p}<0.05$ 
in CAF-like cells which were treated with CM from DLD-1 or HCT-8 (Table 1). Then, the four miRNA levels were further validated in the CAF-like cells induced by four different CRC cells (DLD-1, HCT-8, Lovo, and HCT-116) by qRT-PCR assays. We found that among the four miRNAs, miR-1246, and miR-1290 were significantly overexpressed in the CAF-like cells induced by all four CRC cell lines, compared with CCD-18Co (Figure 1B). Given that miR-1246 was the most upregulated miRNA, we further study the functions of miR-1246 in fibroblasts.

Besides, considering CM from CRC cells could upregulate the expression of miR-1246 in fibroblasts, we reasoned that miR-1246 in fibroblasts could be derived from cancer cells. To validate this hypothesis, HCT- 8 cells were transfected with miR-1246 mimics (Figure 1C). The supernatants were collected after $24 \mathrm{~h}$ and CCD18-Co cells were incubated with the supernatants for another $24 \mathrm{~h}$. As shown in Figure 1D, CCD18-Co cells cultured in CM collected from HCT-8 cells transfected with miR-1246 mimics, had an increased expression level of miR-1246 than the cells cultured in CM from the negative control (NC) group.

In order to study the functional role of miR-1246 in fibroblasts, CCD-18Co cells were transfected with miR-1246 or NC. Overexpression of miR-1246 was verified by qRT-PCR and was significantly elevated in the miR-1246 transfected group, compared with the NC group (Figure 1E). Both NC and miR-1246 groups had high levels of vimentin, a key marker of mesenchymal cells. aSMA, the marker of activated fibroblasts, was significantly upregulated in fibroblasts with ectopic expression of miR-1246, compared with the NC group (Figures 1F, 1G). These results indicated that miR-1246 in cancer cells could upregulate the miR-1246 levels in fibroblasts and activate fibroblasts.

Overexpression of miR-1246 in fibroblasts stimulates cancer cell migration. A previous study showed that CM from CCD-18Co cells could promote the viability of CRC cells [12]. We asked whether the miR-1246 level in CCD-18Co cells could act on CRC cells. To study the impact of miR-1246 overexpressing fibroblasts on cell proliferation and migration of CRC cells, DLD-1 and HCT-8 cells were grown in the presence of CM from CCD-18Co cells transfected with miR-1246 or NC controls. Compared with the CM from the NC group, CM from miR-1246 overexpressing fibroblasts promoted a light increase in cell growth in both DLD-1 and HCT-8 cells (Figure 2A). Intriguingly, CM from the miR-1246 group also led to increased migration in both

Table 1. miRNA expression in CAF-like cells.

\begin{tabular}{lcccc}
\hline & $\begin{array}{c}\text { DLD-1 CM } \\
\text { fold change }^{*}\end{array}$ & p-value & $\begin{array}{c}\text { HCT-8 CM } \\
\text { fold change* }\end{array}$ & p-value \\
\hline hsa-miR-1246 & 4.95 & 0.02 & 38.44 & 0.00 \\
hsa-miR-1268a & 3.46 & 0.02 & 6.34 & 0.03 \\
hsa-miR-4430 & 3.31 & 0.02 & 8.67 & 0.05 \\
hsa-miR-1290 & 3.04 & 0.00 & 13.47 & 0.00 \\
\hline${ }^{*}$ Compared with endogenous miRNA expression levels in CCD-18Co cells.
\end{tabular}

DLD-1 and HCT-8 cells (Figures 2B, 2C). To investigate the loss of function of miR-1246 in fibroblasts, CCD-18Co cells cultured in HCT-8 CM were transfected with the miR-1246 inhibitor or NC control. Knockdown of miR-1246 in the miR-1246 inhibitor group was verified by qRT-PCR (Figure 2D). The CM from the fibroblasts was harvested and HCT- 8 cells were cultured in CM for $24 \mathrm{~h}$. Compared with the CM from the NC group, the CM from the miR-1246 inhibitor group significantly decreased HCT- 8 cell migration (Figures 2E, 2F).

miR-1246 released by fibroblasts is taken up by CRC cells and regulates the Wnt/ $\beta$-catenin pathway in CRC cells. To investigate whether miR-1246 could be taken up by colon cancer cells following release from fibroblasts, GFP-labeled DLD-1 cells were co-cultured indirectly with CCD-18Co cells that were transfected with Cy3-tagged miR-1246. We observed Cy3 red fluorescence in the DLD-1 cells using a confocal microscope (Figure 3A). Furthermore, after incubating DLD- 1 and HCT- 8 cells with the CM from CCD-18Co transfected with miR-1246, the expression of miR-1246 in the recipient cancer cells was increased, compared to the NC group (Figure 3B). Exosomes play an important role in cell communication. Interestingly, we found that when fibroblasts that were transfected with Cy3-1246 were co-cultured with GFP-DLD-1 cells, the exosome inhibitor GW4869 abolished Cy3 red fluorescence in the recipient cells (Figure 3A).

A previous study showed that miR-1246 suppressed the expression of AXIN2 and GSK3 $\beta$, two key members of the Wnt $/ \beta$-catenin pathway in hepatocellular carcinoma [13]. To observe whether miR-1246 could regulate the Wnt pathway in CRC cells, miR-1246 was overexpressed in HCT- 8 cells and the key members of the pathway were detected. As shown in Figures 3C-3E, $\beta$-catenin was upregulated in the miR-1246 group compared with the NC group. In addition, GSK3 $\beta$ and AXIN2, which function as key members of the $\beta$-catenin degradation complex, were repressed in the miR-1246 group (Figures 3D, 3E). These results indicated that miR-1246 released from fibroblasts was transferred into cancer cells and activated the Wnt signaling pathway.

miR-1246 expression level was negatively associated with DFS of CRC patients. To observe the impact of the miR-1246 levels on OS and DFS in CRC patients, we went through the TCGA database. Although Kaplan-Meier survival curves showed that miR-1246 expression level was not associated with OS of CRC patients ( $\mathrm{p}=0.22$, Figure $4 \mathrm{~A})$, patients with a relatively lower expression of miR-1246 had significantly longer disease-free interval (DFI) than those with a higher expression $(\mathrm{HR}=0.38, \mathrm{p}=0.02$, Figure $4 \mathrm{~B})$.

\section{Discussion}

Tumor microenvironment (TME), which consists of fibroblasts, endothelial cells, and immune cells, plays an important role in cancer progression and therapy resistance. 
A

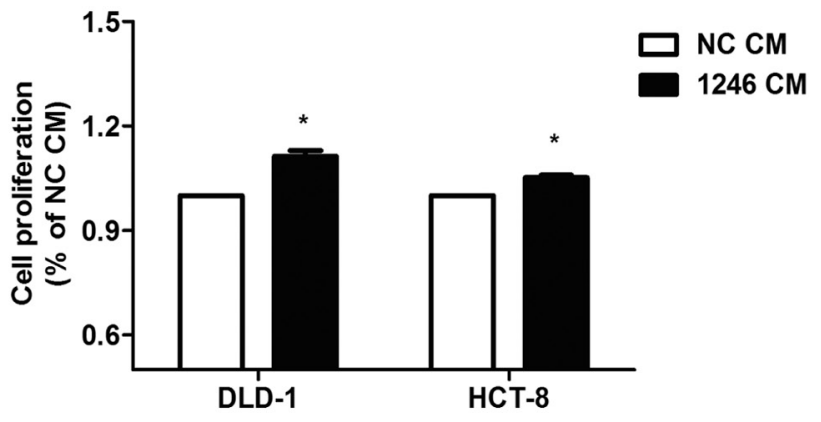

B

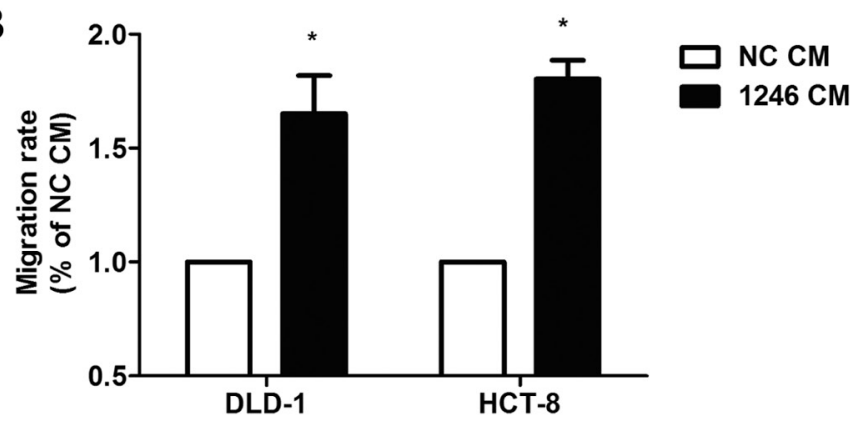

C

NC CM

$1246 \mathrm{CM}$

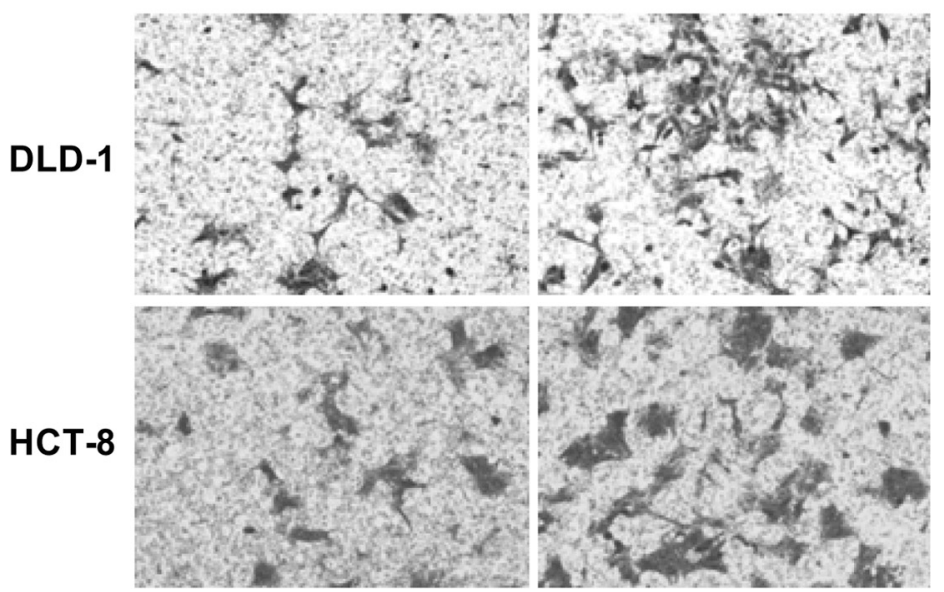

D

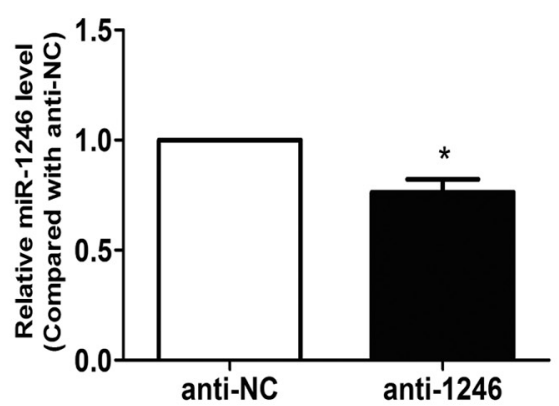

E

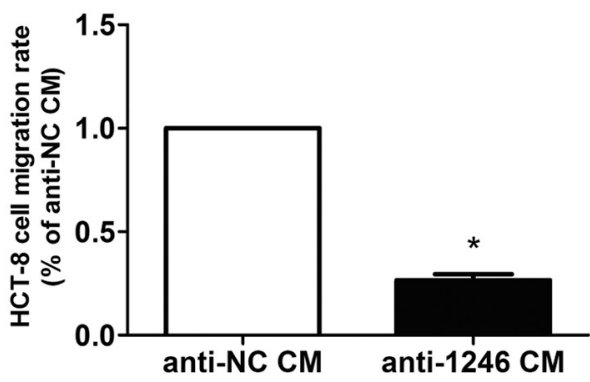

F HCT-8

Anti-NC CM

Anti-1246 CM

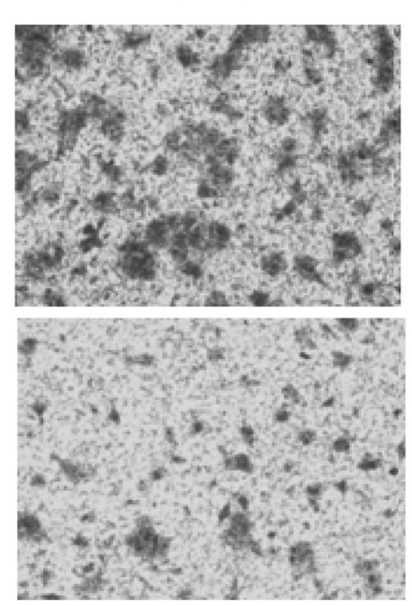

Figure 2. Overexpression of miR-1246 in CCD-18Co promoted proliferation and migration of CRC cells. A) CM from CCD-18Co cells transfected with miR-1246 mimics promoted DLD-1 and HCT-8 cell proliferation compared with CM from CCD-18Co-NC. B, C) CM from CCD-18Co with overexpression of miR-1246 promoted DLD-1 and HCT-8 cell migration compared with CM from CCD-18Co-NC. Data represent the mean \pm SD of three independent experiments. ${ }^{\star} p<0.05$ compared with $\mathrm{CM}$ from the $\mathrm{NC}$ group, respectively. D) miR-1246 expression was repressed in fibroblasts transfected with miR-1246 inhibitor. ${ }^{*} \mathrm{p}<0.05$ compared with miR-1246 levels in the anti-NC group. E, F) CCD-18Co cells were cultured in HCT-8 CM for $48 \mathrm{~h}$ to obtain a CAF phenotype. Cells were transfected with miR-1246 inhibitor or NC. CM from the anti-miR-1246 group inhibited HCT-8 cell migration compared with $\mathrm{CM}$ from the NC group. Data represent the mean $\pm \mathrm{SD}$ of three independent experiments. ${ }^{*} \mathrm{p}<0.05$ compared with $\mathrm{CM}$ from the anti-NC group.

Cancer cells trigger stromal cell-reprogramming towards a cancer-promoting state. The reprogrammed stromal cells promote cancer progression by releasing cytokines, growth factors, and exosomes $[14,15]$. Thus, blocking the cross-talk between cancer cells and stromal cells serves as a poten- tial strategy for cancer therapy [16]. CAFs are an abundant TME component and have been proven to be crucial in cancer development. CAFs have become an attractive target in cancer therapy because of their genome stability and the reduced occurrence of drug resistance. 

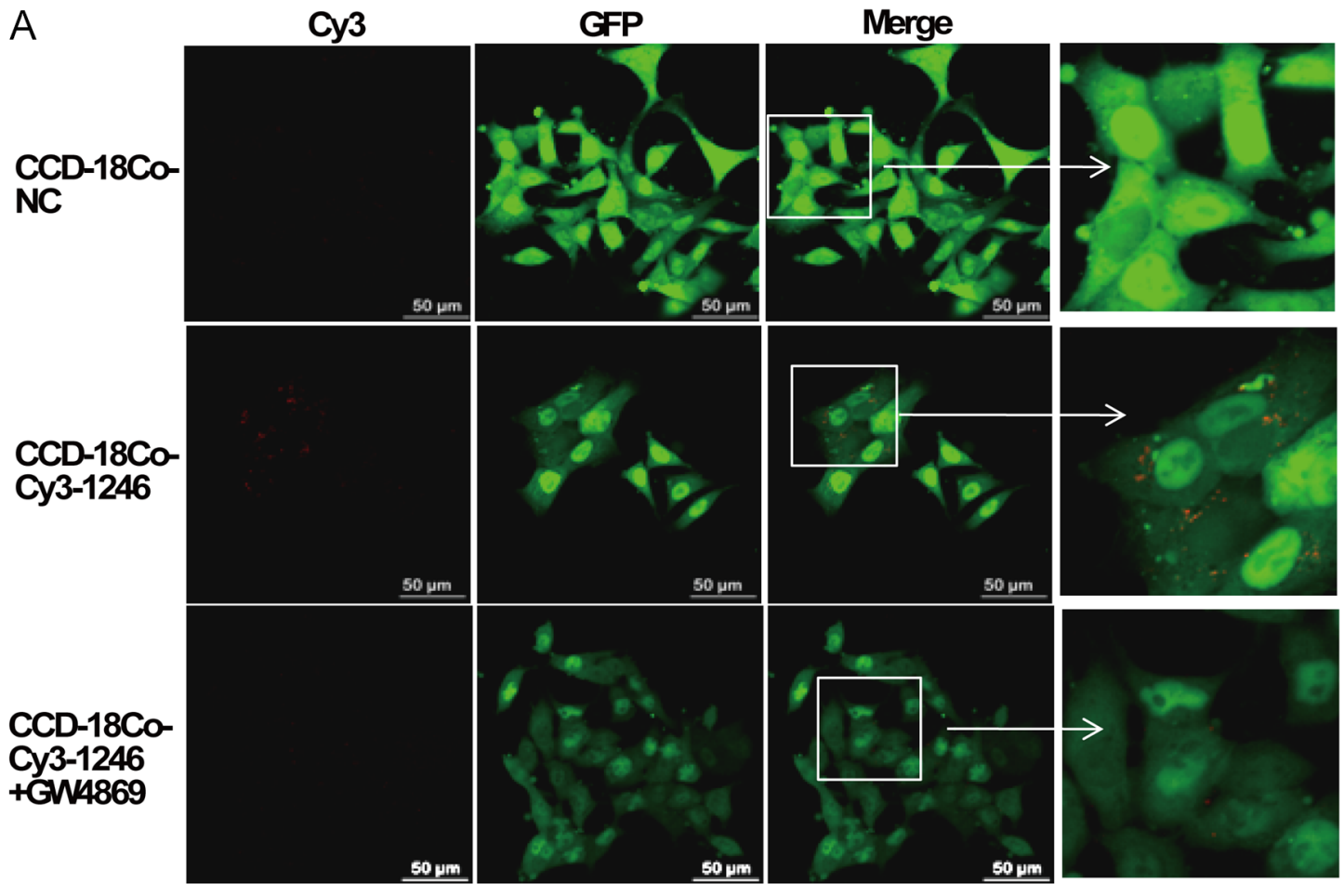

B
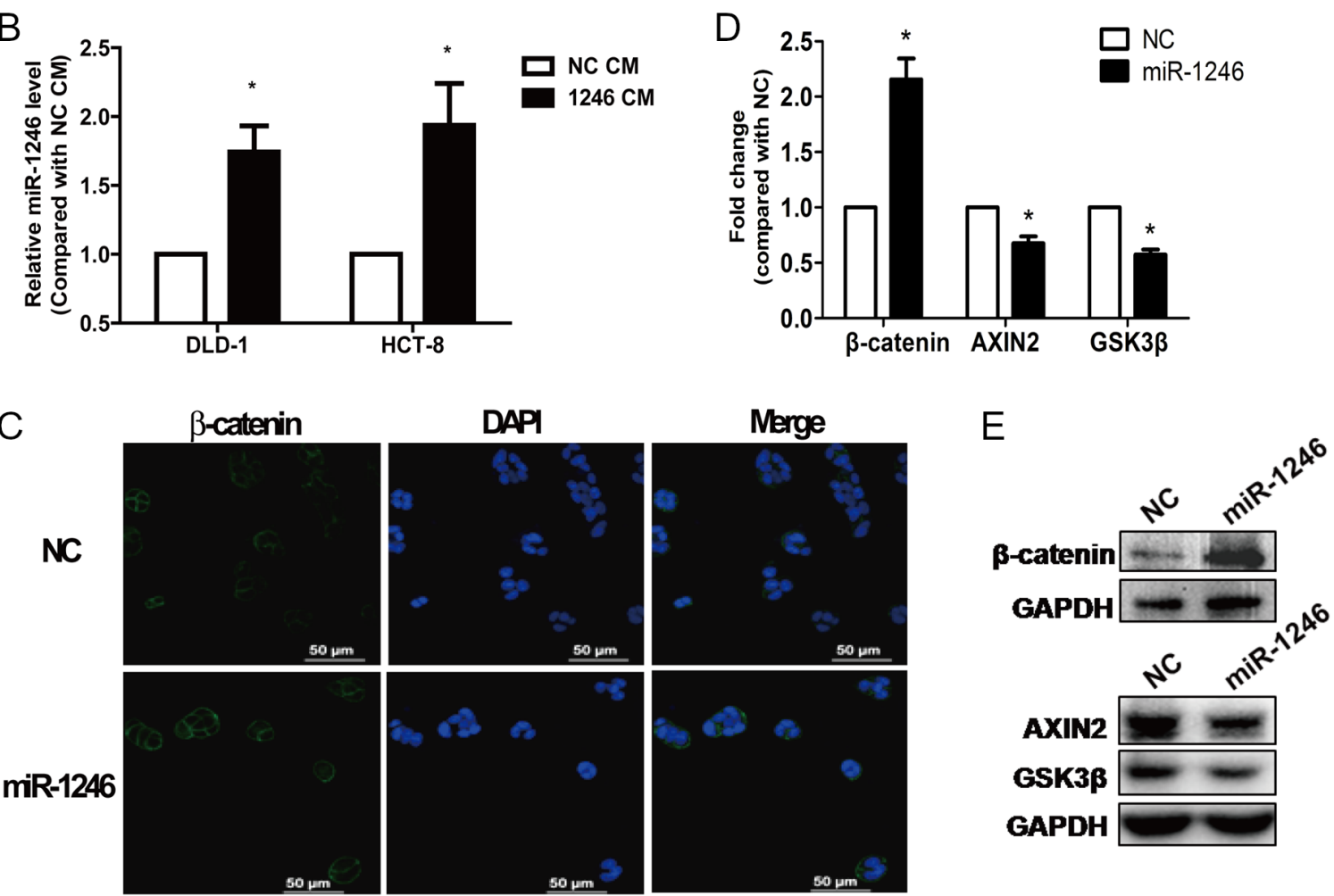

Figure 3. miR-1246 was transferred from fibroblasts to CRC cells and activated the Wnt/ $\beta$-catenin pathway in CRC cells. A) CCD-18Co cells transfected with Cy3-tagged miR-1246 were co-cultured indirectly with GFP-labeled DLD-1. Cy3-miR-1246 was found in DLD-1 cells (630× magnification). CCD-18Co transfected with NC was used as a negative control. B) DLD-1 and HCT-8 cells were cultured in CM from CCD-18Co with overexpression of miR-1246, qRT-PCR analyzes the expression of miR-1246 in the recipient cancer cells. ${ }^{*} \mathbf{p}<0.05$ compared with $\mathrm{CM}$ from the NC group. C) Immunofluorescence analyses of $\beta$-catenin levels in HCT-8 cells transfected with miR-1246 mimics or NC. D, E) Western blot analyses of $\beta$-catenin, AXIN2, and GSK $3 \beta$ levels in HCT- 8 cells with the miR-1246 overexpression. ${ }^{*} \mathbf{p}<0.05$ compared with the NC group. 
In this study, we show that colorectal fibroblast cells CCD-18Co cultured in CM from CRC cells obtain a CAF phenotype and the expression of miR-1246 is upregulated in the CAF-like fibroblasts. miR-1246 derived from cancer cells can be delivered to neighboring fibroblasts, which triggers their reprogramming into a CAF-like state. On the other hand, miR-1246, derived from fibroblasts, enters into colon cancer cells and promotes cancer cell migration by activating the Wnt/ $\beta$-catenin pathway. Additionally, higher expression of miR-1246 is associated with shorter DFI in CRC patients, which implies those patients will develop earlier disease recurrence after surgical resection [17]. However, the expression level of miR-1246 is not associated with OS in CRC patients. It might be due to the treatment for recurrent disease. Collectively, these findings support a cancerpromoting role of miR-1246 in CRCs.

miR-1246 is recognized as a cancer-associated miRNA in a number of different types of cancer. For example, circulating miR-1246 has been used as a potential biomarker for the early diagnosis of gastric cancers and a prognostic biomarker for hepatocellular carcinomas [18, 19]. Additionally, miR-1246 promotes metastasis and invasion of lung cancer cells, by targeting GSK-3 $\beta$ [20]. Also, miR-1246 could regulate CCNG2 to enhance oral squamous cell carcinoma stemness and chemoresistance [21]. Our results indicate that miR-1246 also acts as an oncomir in CRC cells by promoting the Wnt signaling pathway. As distinguished from other studies of miR-1246 functioning in cancer cells, our study sheds new light on the function of miR-1246 in assisting CAFs to regulate CRC.

In conclusion, miR-1246 can shuttle between cancer cells and fibroblasts. The uptake of miR-1246 derived from CRC cells triggers normal fibroblasts reprogramming to the CAF phenotype. On the other hand, miR-1246 secreted by CAFs can be utilized by CRC cells and promotes cell migration (Figure 5). Our study indicates that targeting miR-1246 or blocking its transport from CAFs to CRC cells may represent a promising therapeutic strategy for the treatment of CRCs.

Acknowledgments: This work was supported by the National Natural Science Foundation of China (No.81403238). We thank Joseph Tuersley for linguistic assistance for this paper.

\section{References}

[1] MUELLER MM, FUSENIG NE. Friends or foes - bipolar effects of the tumour stroma in cancer. Nat Rev Cancer 2004; 4: 839-849. https://doi.org/10.1038/nrc1477

[2] SHA M, JEONG S, QIU BJ, TONG Y, XIA L et al. Isolation of cancer-associated fibroblasts and its promotion to the progression of intrahepatic cholangiocarcinoma. Cancer Med 2018; 7: 4665-4677. https://doi.org/10.1002/ cam4.1704
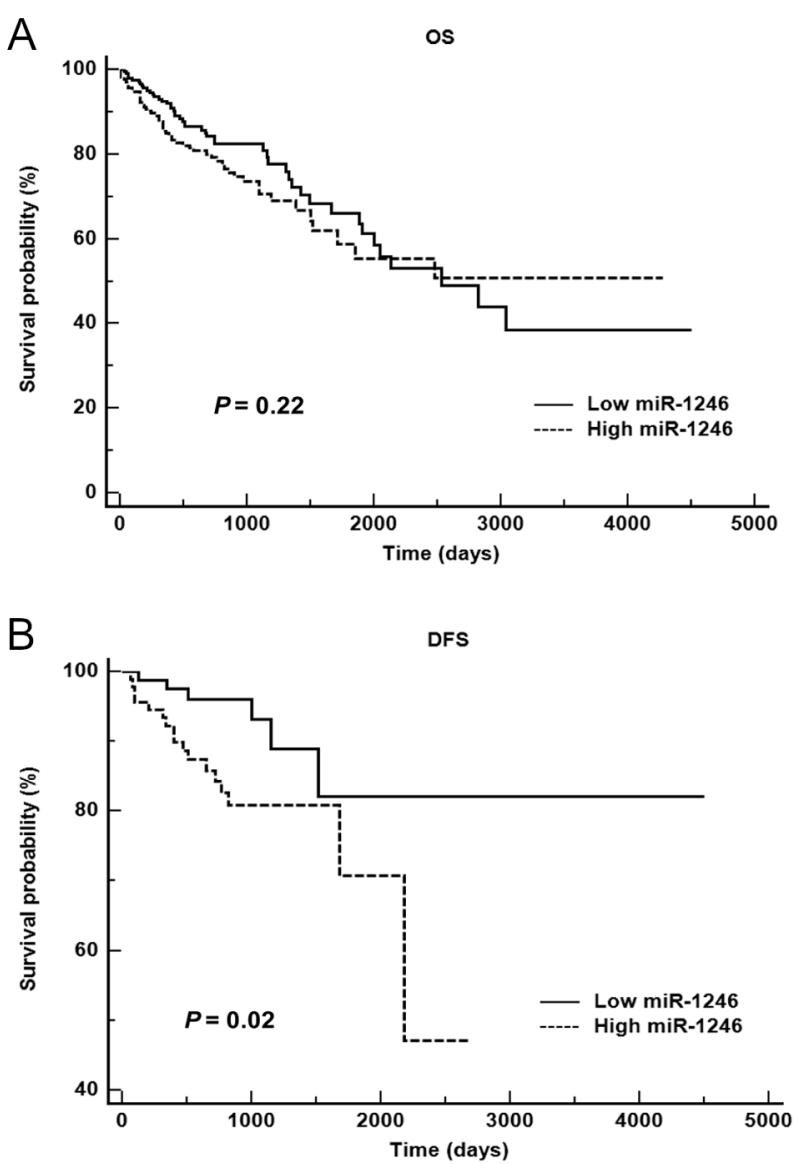

Figure 4. miR-1246 expression was negatively associated with DFS but not OS in CRC patients. A) Kaplan-Meier survival curves showed that the miR-1246 expression level was not associated with OS of CRC patients. Low expression group, $\mathrm{n}=213$, high expression group, $\mathrm{n}=212, \mathrm{p}=\mathbf{0 . 2 2}$. $\mathrm{B}$ ) Kaplan-Meier survival curves showed that low miR-1246 expression significantly associated with better DFS for CRC patients $(\mathrm{HR}=0.38,95 \%$ CI: 0.17-0.85, p=0.02). Significance was determined using the log rank test. Low expression group, $\mathrm{n}=\mathbf{8 4}$, high expression group, $\mathrm{n}=\mathbf{9 2}$.

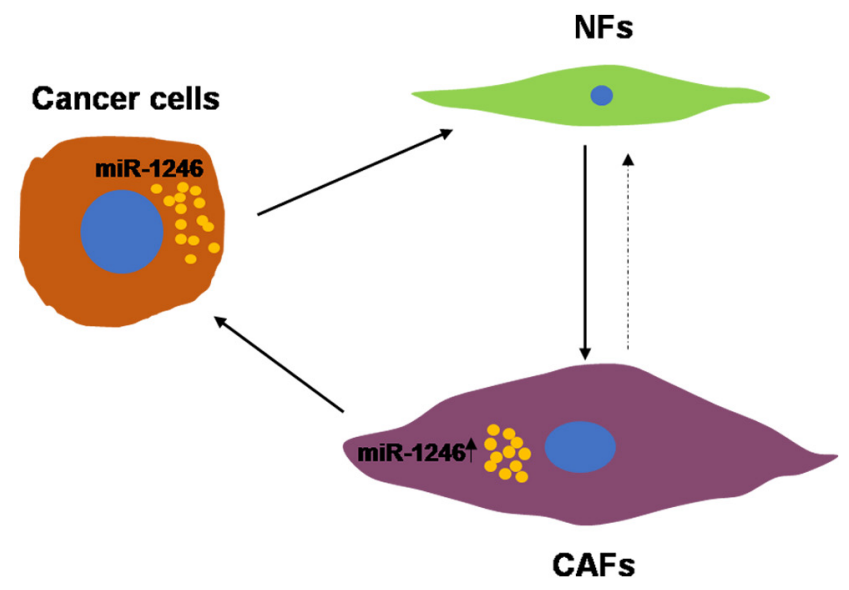

Figure 5. Diagram shows miR-1246 shuttles between fibroblasts and CRC cells to promote CRC cell migration. 
[3] ORIMO A, GUPTA PB, SGROI DC, ARENZANA-SEISDEDOS F, DELAUNA YT et al. Stromal fibroblasts present in invasive human breast carcinomas promote tumor growth and angiogenesis through elevated SDF-1/CXCL12 secretion. Cell 2005; 121: 335-348. https://doi.org/10.1016/j. cell.2005.02.034

[4] ROZENCHAN PB, CARRARO DM, BRENTANI H, DE CARVALHO MOTA LD, BASTOS EP et al. Reciprocal changes in gene expression profiles of cocultured breast epithelial cells and primary fibroblasts. Int J Cancer 2009; 125: 2767-2777. https://doi.org/10.1002/ijc.24646

[5] GARUFI A, TRAVERSI G, CIRONE M, D’ORAZI G. HIPK2 role in the tumor-host interaction: Impact on fibroblasts transdifferentiation CAF-like. IUBMB Life 2019; 71: 20552061. https://doi.org/10.1002/iub.2144

[6] BARTEL DP. MicroRNAs: target recognition and regulatory functions. Cell 2009; 136: 215-233. https://doi.org/10.1016/j. cell.2009.01.002

[7] SHEN H, YU X, YANG F, ZHANG Z, SHEN J et al. Reprogramming of Normal Fibroblasts into Cancer-Associated Fibroblasts by miRNAs-Mediated CCL2/VEGFA Signaling. PLoS Genet 2016; 12: e1006244. https://doi.org/10.1371/ journal.pgen.1006244

[8] MITRA AK, ZILLHARDT M, HUA Y, TIWARI P, MURMANN AE et al. MicroRNAs reprogram normal fibroblasts into cancer-associated fibroblasts in ovarian cancer. Cancer Discov 2012; 2: 1100-1108. https://doi.org/10.1158/21598290.CD-12-0206

[9] CHATTERJEE A, JANA S, CHATTERJEE S, WASTALL LM, MANDAL $G$ et al. MicroRNA-222 reprogrammed cancerassociated fibroblasts enhance growth and metastasis of breast cancer. Br J Cancer 2019; 121: 679-689. https://doi. org/10.1038/s41416-019-0566-7

[10] LOPEZ-ROMERO P. Pre-processing and differential expression analysis of Agilent microRNA arrays using the AgiMicroRna Bioconductor library. BMC Genomics 2011; 12: 64. https://doi.org/10.1186/1471-2164-12-64

[11] CUI F, WANG S, LAO I, ZHOU C, KONG H et al. miR-375 inhibits the invasion and metastasis of colorectal cancer via targeting SP1 and regulating EMT-associated genes. Oncol Rep 2016; 36: 487-493. https://doi.org/10.3892/or.2016.4834

[12] ZHOU W, XU G, WANG Y, XU Z, LIU X et al. Oxidative stress induced autophagy in cancer associated fibroblast enhances proliferation and metabolism of colorectal cancer cells. Cell Cycle 2017; 16: 73-81. https://doi.org/10.1080/153 84101.2016 .1252882
[13] CHAI S, NG KY, TONG M, LAU EY, LEE TK et al. Octamer 4/microRNA-1246 signaling axis drives Wnt/beta-catenin activation in liver cancer stem cells. Hepatology 2016; 64: 2062-2076. https://doi.org/10.1002/hep.28821

[14] DENG X, RUAN H, ZHANG X, XU X, ZHU Y et al. Long noncoding RNA CCAL transferred from fibroblasts by exosomes promotes chemoresistance of colorectal cancer cells. Int J Cancer 2020; 146: 1700-1716. https://doi.org/10.1002/ ijc.32608

[15] COOKS T, PATERAS IS, JENKINS LM, PATEL KM, ROBLES AI et al. Mutant p53 cancers reprogram macrophages to tumor supporting macrophages via exosomal miR-1246. Nat Commun 2018; 9: 771. https://doi.org/10.1038/s41467018-03224-w

[16] CALON A, LONARDO E, BERENGUER-LLERGO A, ESPINET E, HERNANDO-MOMBLONA $\mathrm{X}$ et al. Stromal gene expression defines poor-prognosis subtypes in colorectal cancer. Nat Genet 2015; 47: 320-329. https://doi. org/10.1038/ng.3225

[17] HOPPENER D J, NIEROP P M H, VAN AMERONGEN M J, OLTHOF P B, GALJART B et al. The Disease-Free Interval Between Resection of Primary Colorectal Malignancy and the Detection of Hepatic Metastases Predicts Disease Recurrence But Not Overall Survival. Ann Surg Oncol 2019; 26: 2812-2820. https://doi.org/10.1245/s10434-019-07481-x

[18] SHI Y, WANG Z, ZHU X, CHEN L, MA Y et al. Exosomal miR-1246 in serum as a potential biomarker for early diagnosis of gastric cancer. Int J Clin Oncol 2020; 25: 89-99. https://doi.org/10.1007/s10147-019-01532-9

[19] CHUMA M, TOYODA H, MATSUZAKI J, SAITO Y, KUMADA T, et al. Circulating microRNA-1246 as a possible biomarker for early tumor recurrence of hepatocellular carcinoma. Hepatol Res 2019; 49: 810-822. https://doi. org/10.1111/hepr.13338

[20] YANG F, XIONG H, DUAN L, LI Q, LI X et al. MiR-1246 Promotes Metastasis and Invasion of A549 cells by Targeting GSK-3betaMediated Wnt/beta-Catenin Pathway. Cancer Res Treat 2019; 51: 1420-1429. https://doi.org/10.4143/ crt.2018.638

[21] LIN SS, PENG CY, LIAO YW, CHOU MY, HSIEH PL et al. miR-1246 Targets CCNG2 to Enhance Cancer Stemness and Chemoresistance in Oral Carcinomas. Cancers (Basel) 2018; 10: 272. https://doi.org/10.3390/cancers 10080272 